\title{
Racial and Regional Disparities in Outcomes Among Veterans Initially Adherent to Oral Antidiabetic Therapies: an Observational Cohort Study
}

\author{
Justin Gatwood, $P h D^{1,2}$ (1) , Marie Chisholm-Burns, PharmD ${ }^{7}$, Robert Davis, $\mathrm{MD}^{3}$, \\ Fridtjof Thomas, $P h D^{4}$, Praveen Potukuchi, $M S^{5}$, Adriana Hung, MD ${ }^{6,7}$, and \\ Csaba P. Kovesdy, MD 5,8
}

\begin{abstract}
'University of Tennessee Health Science Center, College of Pharmacy, Memphis, TN, USA; ${ }^{2}$ Department of Clinical Pharmacy, University of Tennessee College of Pharmacy, Nashville, TN, USA; ${ }^{3}$ University of Tennessee Health Science Center, Center for Biomedical Informatics, Memphis, TN, USA; ${ }^{4}$ University of Tennessee Health Science Center, Department of Preventive Medicine, Memphis, TN, USA; ${ }^{5}$ Division of Nephrology, University of Tennessee Health Science Center, Memphis, TN, USA; ${ }^{6}$ Vanderbilt University School of Medicine, Nashville, TN, USA; ${ }^{7}$ VA Tennessee Valley Healthcare System, Nashville, TN, USA; ${ }^{8}$ Memphis VA Medical Center, Memphis, TN, USA
\end{abstract}

BACKGROUND: Adherence to prescribed medications is connected with, but is not a guarantee of, improved disease management and health outcomes. It remains unclear whether underlying health disparities exist among patients adherent to therapy and whether differences in outcomes vary by race and residential areas of the country.

OBJECTIVE: To determine the extent of racial and regional variation in outcomes within 5 years of oral antidiabetic drug initiation among veterans adherent to therapy.

DESIGN: Retrospective cohort study of 83,265 US Veterans Health Administration data, 2002-2014

PATIENTS: US veterans with uncomplicated diabetes and taking oral antidiabetic agents

MAIN MEASURES: Veterans initially adherent to oral antidiabetic therapy were followed for up to 5 years, and comparisons focused on differences between nonHispanic White and non-Hispanic Black veterans across geographic region and residential type (urban or rural). Outcomes included composite cardiovascular events, composite cerebrovascular events, or all-cause mortality using Poisson and adjusted Cox proportional hazards models.

KEY RESULTS: Cardiovascular event and all-cause mortality rates differed by race and region, while urban/rural differences were evident for cerebrovascular events and all-cause mortality. For non-Hispanic Blacks, the mortality rate was half that compared to non-Hispanic Whites (6.5 [95\% CI 5.8-7.2] versus 13.3 [95\% CI 12.9-13.8], $p<0.0001)$. Compared to the Northeast, all other regions had higher adjusted hazards for cardiovascular or cerebrovascular events (with a single exception), but no regional differences in all-cause mortality were observed. Models with interactions demonstrated that racial differences in cardiovascular events and all-cause mortality were isolated to the Midwest (HR 1.99 [95\% CI 1.301-

Electronic supplementary material The online version of this article (https://doi.org/10.1007/s11606-019-05373-0) contains supplementary material which is available to authorized users.

Prior Presentations Results described in this manuscript were present ed as a poster and podium at the 2019 American College of Clinical Pharmacu Annual Meetina in Seattle. WA March 2019).

Received May 17, 2019

Accepted September 12, 2019

Published online January 8, 2020
3.06; HR 1.64 [95\% CI 1.210-2.215]) and South (HR 1.69 [85\% CI $1.347-2.131$ ]; HR 1.27 [95\% CI $1.095-$ $1.470])$.

CONCLUSIONS: Despite adherence to therapy, differences in outcomes are likely among veterans with diabetes based on race and geography. Localized analyses may uncover specific social determinants contributing to differences in outcomes.

KEY WORDS: diabetes; adherence; regional variation; racial disparities.

J Gen Intern Med 35(4):1211-8

DOI: $10.1007 / \mathrm{s} 11606-019-05373-0$

(C) Society of General Internal Medicine 2020

\section{INTRODUCTION}

The role of medication adherence in managing diabetes is well established: compared to those intermittently or continuously foregoing treatment, patients adhering to prescribed regimens tend to experience greater reductions in hemoglobin $\mathrm{A} 1 \mathrm{C}$ and are more likely to achieve recommended treatment goals. ${ }^{1-11}$ Furthermore, achieving particular adherence thresholds, such as a proportion of days covered (PDC) of at least $80 \%$, has been used to compare long-term outcomes between adherent and nonadherent patients. In analyses ranging from 3 to 9 years of follow-up, being adherent has been connected with a decreased likelihood of diabetes-related outcomes, such as hypoglycemia, microvascular complications, hospitalization, and cardiovascular or cerebrovascular events. ${ }^{12-16}$

Despite comparatively better outcomes by having more medication on hand (i.e., higher PDC), being adherent to antidiabetic medications is not a guarantee that undesired outcomes will be avoided. Our previous analyses comparing cardiovascular, cerebrovascular, microvascular, and all-cause mortality outcomes by medication adherence status among veterans with diabetes revealed that while many outcomes were improved by higher rates of medication use, adherence did not prevent all targeted outcomes. ${ }^{12}$ Moreover, when adjusting for patient characteristics 
and first-year medication adherence, differences in outcomes were observed in subgroups of this population, including minorities and those residing in different geographical regions. Such findings reinforce the importance of adherence while suggesting the need for deeper analyses focusing on potential disparities among population subgroups.

A key challenge in determining whether disparities exist is in isolating factors influencing disparate outcomes. Within the USA, access to care, including living near accessible healthcare providers or having reliable means of obtaining prescribed therapies, is a potential barrier to improved health outcomes for many Americans, particularly minorities, lower income individuals, and those in rural areas. ${ }^{17-19}$ However, within the Veterans Health Administration measures have been taken to improve access to healthcare providers and medications. For instance, the vast majority of outpatient prescriptions are managed by the Veterans Affairs (VA) Mail Order Pharmacy, which delivers medications to veterans irrespective of location. To address the needs of rural veterans and those living distant to a VA medical center, the VA established community-based outpatient clinics (CBOCs) throughout the country to provide common outpatient services, such as primary and mental health care, closer to home. Importantly, CBOCs demonstrate comparable performance measures to larger VA medical centers. ${ }^{20}$ In addressing their patients' access to care, the VA provides a unique opportunity to study a population for whom healthcare access may be smaller barriers to improved disease management and outcomes. Furthermore, by isolating those adherent to therapy within a population of veterans, we lessen yet another barrier to improved health outcomes, facilitating a closer look at subgroups to better ascertain factors potentially influencing disparate outcomes.

This study was designed to identify the extent to which health outcomes differ by race and residence among US veterans with diabetes who were initially adherent to their oral antidiabetic (OAD) regimen. Comparisons focused on differences in outcomes between non-Hispanic Black and White veterans, across regions of the USA, and between urban and rural residents. We hypothesized that non-Hispanic Blacks, those residing in the South, and rural residents would have worse outcomes among veterans with uncomplicated diabetes who were initially adherent to antidiabetic therapy.

\section{METHODS}

\section{Study Population and Data}

This was a retrospective cohort study using the VA Corporate Data Warehouse from 2002 through 2014, which included the VA Decision Support System National Data Extracts, Inpatient and Outpatient Medical SAS Datasets, and Vital Status Files. ${ }^{21}$ Institutional review boards at the University of Tennessee Health Science Center and the Memphis VA Medical Center approved this study.
To be included, patients must have been at least 18 years old, been diagnosed with uncomplicated diabetes (ICD-9-CD codes: 250.00 or 250.02) for the first time (i.e., no diabetes diagnostic codes in the year before the initial diabetes diagnosis), been prescribed an OAD for the first time (i.e., no OAD fills in the year before the initial diabetes diagnosis), and have at least 1 year of data prior to their diabetes diagnosis and following OAD initiation. Patients were excluded if they were insulin dependent or had been diagnosed with a diabetesrelated microvascular complication prior to or in conjunction with their diabetes diagnosis. To avoid treatment complexity and potential toxicities impacting overall medication use, patients were also excluded if they were diagnosed with HIV at any time or been diagnosed with malignant cancer prior to their diabetes diagnosis. The resulting cohort was assessed for OAD use over the first 365 days following the initial OAD fill (Appendix 1).

\section{Exposures, Outcomes, and Covariates}

The main exposure was OAD adherence in the first year of therapy using the PDC metric, which is the preferred medication adherence measure using claims data according to the Pharmacy Quality Alliance. ${ }^{22}$ The numerator was a sum of days supply within each patient's pharmacy records to determine the amount of medication on hand, while adjusting for overlapping days between fills and changes in therapy. The denominator was 365 days. Those achieving a PDC $\geq 80 \%$ in the first year were considered adherent. ${ }^{22}$ Although medication use is an ongoing, potentially dynamic behavior, our earlier analyses indicated that initial medication adherence is highly predictive of future use $\mathrm{e}^{23}$; consequently, initial use patterns are likely to continue, making first-year adherence an appropriate mechanism to subgroup patients in analyzing outcomes.

Discrete events and death by any cause were evaluated for up to 5 years following OAD initiation (follow-up time was censored at the latest 5 years after OAD initiation). Composite cardiovascular (CAD) and cerebrovascular (CVD) events (identified by ICD-9 and current procedural terminology [CPT] codes) included acute myocardial infarction or angina and ischemic stroke or transient ischemic attack, respectively (Appendix 2). Patient death was determined by VA vital status files, which corroborates death using records from VA resource utilization, the Social Security Administration, and the Centers for Medicare and Medicaid Services.

In line with the main objective, the primary predictors were self-reported race/ethnicity and place of residence as documented in each veteran's medical records. Nationwide comparisons were first made between non-Hispanic White and non-Hispanic Black veterans, region of residence (Northeast, Midwest, South, or West by FIPS codes), and population density (rural or urban) while controlling for available patient characteristics extracted at or near OAD initiation. Subsequent analyses compared within and across regions of the country. 
Because this is an exploratory data analysis, we did not adjust for multiplicity in testing and provided $p$ values and confidence intervals are unadjusted for multiplicity in testing.

To control for potential confounding, demographic and clinical characteristics were extracted from available records. Demographic variables included age, sex, marital status, and income (median values by zip code). ${ }^{24}$ Health status was determined using recorded clinical values and derived factors. The Deyo-Charlson Comorbidity Index (CCI) controlled for the presence of diagnoses or events prior to and excluding the initial diabetes diagnosis. ${ }^{25}$ Clinical covariates included body mass index (BMI), baseline hemoglobin A1C ( \pm 90 days of the first $\mathrm{OAD}$ fill), baseline blood pressure values (categorized by JNC 7 hypertension stage), and laboratory values indicating low-density lipoprotein levels (LDL), at or near the first OAD fill ( \pm 90 days). ${ }^{26}$ Associated events and diagnoses of interest occurring prior to the first OAD fill were accounted for in each associated model using ICD-9 and CPT codes. The initial OAD and concomitant use of antihypertensive and/or lipid-lowering therapies (as of the first year of OAD use) were also extracted.

\section{Statistical Analyses}

Patient characteristics were compared descriptively using $t$ tests or chi-squared tests. Poisson models tested for differences in event rates (per 1000 patient-years) while accounting for variable follow-up time. Adjusted Cox proportional hazards models examined differences in relative risk for outcomes and proportionality was confirmed using the standardized score process over 1000 data simulations. ${ }^{27}$ A Cox model also estimated all-cause death hazards when explicitly acknowledging participants had to survive at least 1 year to be included (i.e., late entry into the risk set with first year follow-up not included as study time). The resulting estimates showed no changes of practical importance (not shown); therefore, we are not concerned that estimates suffer from immortal time bias due to the 1-year follow-up requirement. Note that survived cardiovascular and cerebrovascular events are included during that first year, conditional on 1-year survival. Main effects of race/ethnicity, region, and population density were determined by individual models followed by interaction effects to examine variations in outcomes among subgroups of the overall cohort. All analyses were conducted using SAS Enterprise Guide version 7.1 (SAS, Cary, NC) and a statistical significance level of $\alpha=0.05$ was set a priori as the threshold to claim a "real difference."

\section{RESULTS}

\section{Patient Population}

A total of 83,265 patients were included, the majority of which (83.7\%) were non-Hispanic White (Table 1). Comparatively, non-Hispanic Black veterans with diabetes tended to be younger, be unmarried, live in lower income and urban areas, and reside in the South. This group also tended to have higher A1C, blood pressure, and LDL values at baseline (all $p<0.0001$ ). Patient characteristics by region were similar except for a majority of Midwest residents living in rural areas (characteristics by region not shown). Characteristics by population density were also mostly similar, but rural veterans tended to live in lower income zip codes $(p<0.0001)$, have slightly higher baseline CCI $(0.48$ vs. $0.43 ; p<0.0001)$ and BMI values (33.3 vs. $32.9 ; p<0.0001)$, and report being married $(64.6 \%$ vs. $55.2 \% ; p<0.0001)$.

\section{Event Rates}

Few cardiovascular or cerebrovascular events were observed within the first 5 years of OAD use. Non-Hispanic Whites had higher unadjusted rates (per 1000 patient-years) of composite CAD events $(4.8$ versus $2.8, p<0.0001)$ and all-cause death (13.3 versus $6.5, p<0.0001)$. Across regions, the Northeast had the lowest CAD event rates but the highest all-cause mortality rate. Rural veterans had lower rates of composite CVD events ( 1.7 versus 2.1 per 1000 patient-years, $p<0.01$ ), but higher rates of all-cause death (12.7 versus 11.8 per 1000 patient-years, $p<0.05$ ) (Table 2).

\section{Adjusted Event Hazards}

Northeast residents had better or comparable CAD and CVD outcomes compared to other regions, but no significant variation in all-cause mortality was observed across regions. Rural-dwelling veterans had lower adjusted CVD hazards (HR 0.83; 95\% CI 0.702-0.977); however, no significant differences were observed for either CAD or all-cause mortality. Similar to unadjusted rate analyses, non-Hispanic Blacks had lower adjusted hazards for composite CAD events (HR 0.62; 95\% CI 0.516-0.742) and all-cause death (HR 0.77; 95\% CI 0.683-0.865) compared to non-Hispanic Whites (Fig. 1).

\section{Regional Variation}

Relatively few differences across region were observed among non-Hispanic Black veterans, but all-cause mortality in the Midwest was comparatively worse than the Northeast (HR $1.60 ; 95 \%$ CI 1.042-2.443) and the South (HR 0.70; 95\% CI 0.509-0.971). Regional variation among non-Hispanic White veterans followed the same pattern as the full model without interactions. When considering population density (urban/rural), those residing in the Northeast had lower CAD and CVD hazards in the majority of regional comparisons irrespective of residential area. The only other significant difference for these outcomes included residents of the South demonstrating a lower CAD event hazard (HR 0.81; 95\% CI 0.678-0.974) compared to the West. Additionally, all-cause mortality was lower for urban veterans in the Midwest (HR 0.91; 95\% CI 0.836-0.985) versus the South, and those in the rural 
Table 1 Patient Demographic and Clinical Characteristics

\begin{tabular}{|c|c|c|c|c|}
\hline Characteristic* & All $(n=83,265)$ & Non-Hispanic White $(n=69,679)$ & Non-Hispanic Black $(n=13,586)$ & $p$ values \\
\hline Age, mean, (SD) & $62.5(10.4)$ & $63.6(10.2)$ & $57.1(9.8)$ & $\begin{array}{l}< \\
0.0001\end{array}$ \\
\hline \multicolumn{5}{|l|}{ Age group, no. (\%) } \\
\hline$<35$ & $419(0.5)$ & $285(0.4)$ & $134(1.0)$ & $<$ \\
\hline $35-44$ & $3542(4.3)$ & $2318(3.3)$ & $1224(9.0)$ & 0.0001 \\
\hline $45-54$ & $13,271(15.9)$ & $9045(13.0)$ & $4226(31.1)$ & \\
\hline $55-64$ & $35,824(43.0)$ & $30,243(43.4)$ & $5581(41.1)$ & \\
\hline $65-74$ & $19,310(23.2)$ & $17,543(25.2)$ & $1767(13.0)$ & \\
\hline $75-84$ & 9619 (11.6) & $9032(13.0)$ & $587(4.3)$ & \\
\hline $85+$ & $1280(1.5)$ & $1213(1.7)$ & $67(0.5)$ & \\
\hline Male, no. $(\%)$ & $79,989(96.1)$ & $67,466(96.8)$ & $12,523(92.2)$ & $\stackrel{<}{0.0001}$ \\
\hline \multicolumn{5}{|l|}{ Marital status, no. $(\%)^{\dagger}$} \\
\hline Married & $48,726(58.5)$ & $42,438(60.9)$ & $6288(46.3)$ & $<$ \\
\hline Divorced/separated & $20,678(24.8)$ & $16,128(23.1)$ & $4550(33.5)$ & 0.0001 \\
\hline Single/never married & $6387(7.7)$ & $4588(6.6)$ & 1799 (13.2) & \\
\hline \multirow{2}{*}{\multicolumn{5}{|c|}{ Geographic region, no. $(\%)^{\dagger}$}} \\
\hline & & & & \\
\hline Northeast & $10,906(13.1)$ & 9654 (13.9) & $1252(9.2)$ & $<$ \\
\hline Midwest & $21,176(25.4)$ & $18,855(27.1)$ & $2321(17.1)$ & 0.0001 \\
\hline South & $37,132(44.6)$ & $28,507(40.9)$ & $8625(63.5)$ & \\
\hline West & $13,769(16.5)$ & $12,431(17.8)$ & $1338(9.8)$ & \\
\hline \multicolumn{5}{|l|}{ Population density, no. $(\%)^{\dagger}$} \\
\hline Urban & $47,498(57.0)$ & $36,447(52.3)$ & $11,051(81.3)$ & \\
\hline Rural & $35,483(42.6)$ & $32,997(47.4)$ & $2486(18.3)$ & \\
\hline \multicolumn{5}{|l|}{ Median income, no. (\%) } \\
\hline$<50,000$ & $50,236(60.3)$ & $41,063(58.9)$ & $9173(67.5)$ & $<$ \\
\hline $50,000-75,000$ & $26,010(31.2)$ & $22,496(32.3)$ & $3514(25.9)$ & 0.0001 \\
\hline$>75,000$ & $7019(8.4)$ & $6120(8.8)$ & $899(6.6)$ & \\
\hline \multicolumn{5}{|l|}{ Initial antidiabetic medication, no. (\%) } \\
\hline Metformin & $54,016(64.9)$ & $45,776(65.7)$ & $8240(60.7)$ & $<$ \\
\hline Sulfonylurea & $26,429(31.7)$ & $21,590(31.0)$ & $4839(35.6)$ & 0.0001 \\
\hline Thiazolidinedione & $1805(2.2)$ & $1486(2.1)$ & $319(2.3)$ & \\
\hline All others & $1015(1.2)$ & $827(1.2)$ & $188(1.4)$ & \\
\hline Body mass index, mean (SD) & $33.1(6.0)$ & $33.2(5.9)$ & $32.7(6.0)$ & $\begin{array}{l}< \\
0.0001\end{array}$ \\
\hline Hemoglobin A1C, mean (SD), \% & $7.4(1.6)$ & $7.3(1.5)$ & $7.8(1.9)$ & $\begin{array}{l}< \\
0.0001\end{array}$ \\
\hline \multicolumn{5}{|l|}{ Blood pressure, mean (SD), mm Hg } \\
\hline Systolic & $134.3(16.8)$ & $134.2(16.7)$ & $135.0(17.4)$ & $\begin{array}{l}< \\
0.0001\end{array}$ \\
\hline Diastolic & $77.5(10.9)$ & $76.9(10.8)$ & $80.3(11.3)$ & $\begin{array}{l}< \\
0.0001\end{array}$ \\
\hline $\begin{array}{l}\text { Cholesterol, mean (SD), mg/dL } \\
\text { LDL }\end{array}$ & $96.4(34.9)$ & $94.7(34.2)$ & $104.8(37.1)$ & \\
\hline HDL & $39.0(11.1)$ & $38.3(10.6)$ & $42.6(12.8)$ & $\begin{array}{l}0.0001 \\
< \\
0.0001\end{array}$ \\
\hline Charlson Comorbidity Index, mean (SD) ${ }^{*}$ & $0.45(0.65)$ & $0.47(0.66)$ & $0.35(0.57)$ & $\begin{array}{l}0.0001 \\
< \\
0.0001\end{array}$ \\
\hline
\end{tabular}

*Characteristics prior to starting OAD therapy. Proportions may not sum to $100 \%$ owing to rounding and missing data † Missing values: 999 (marital status), 282 (region), 284 (density)

Excludes qualifying diabetes diagnosis

Northeast had a higher adjusted hazard for all-cause mortality versus the rural Midwest (HR 1.17; 95\% CI 1.003-1.352).

\section{Subgroup Analyses}

Within race, no differences in outcomes were observed between urban and rural residents. However, analyses by population density confirmed consistently lower hazards for CAD events and all-cause mortality for non-Hispanic Black veterans. Within geographic region, the comparatively worse adjusted hazards for CAD events and all-cause mortality by race resided solely in the Midwest and South. Additionally, in spite of a lack of main effect by race, non-Hispanic White veterans in the West had a lower hazard for CVD outcomes
(HR 0.60; 95\% CI 0.366-0.967) when compared to nonHispanic Black veterans in the same region. The observed main effect by population density for CVD outcomes appeared to be driven, at least in part, by lower hazards among ruraldwelling veterans in the Northeast (HR 0.51; 95\% CI 0.2910.899) and West (HR 0.66; 95\% CI 0.435-0.986) (Fig. 2).

\section{CONCLUSIONS}

This study used a novel approach to investigate how outcomes among veterans with diabetes varied by region, race, and population density to identify subgroups in which underlying diabetes-related disparities may exist. Importantly, the analysis 
Table 2 Unadjusted Event Rates Within 5 Years of Oral Antidiabetic Medication Initiation

\begin{tabular}{llll}
\hline \hline Category & Cardiovascular event & Cerebrovascular event & All-cause death \\
\hline Race & & & $13.3(12.9-13.8)$ \\
$\quad$ Non-Hispanic White & $4.8(4.50-5.01)$ & $1.9(1.79-2.11)$ & $6.5(5.80-7.20)^{* *}$ \\
$\quad$ Non-Hispanic Black & $2.8(2.36-3.24)^{*}$ & $2.1(1.79-2.56)$ & $14.2(13.2-15.3)$ \\
Region & $3.0(2.58-3.59)$ & $1.5(1.20-1.92)$ & $12.7(12.0-13.5)^{*}$ \\
$\quad$ Northeast & $4.9(4.46-5.39)^{* *}$ & $1.9(1.60-2.17)$ & $1.7(11.1-12.2)^{* *}$ \\
Midwest & $4.4(4.09-4.75)^{* *}$ & $2.0(1.83-2.28)^{*}$ & $11.0(10.2-11.9)^{* *}$ \\
South & $4.8(4.26-5.40)^{* *}$ & $2.2(1.89-2.67)^{*}$ & $11.8(11.3-12.3)$ \\
$\quad$ West & $4.3(4.00-4.50)$ & $2.1(1.90-2.35)$ & $12.7(12.2-13.3)^{*}$ \\
Population density & $4.6(4.30-5.00)$ & $1.7(1.52-1.94)^{*}$ & \\
$\quad$ Urban & & & 12.7 \\
Rural & &
\end{tabular}

Values listed are per 1000 person-years (95\% confidence interval). Within category comparisons identifying significant differences to reference rate (first row of each category): $* p<0.05 ; * * p<0.0001$. Years of follow-up time (mean [SD]): cardiovascular event (4.2 [1.16]); cerebrovascular event (4.2 [4.18]); all-cause death (4.2 [1.12])

only included those initially adherent to OAD therapy, limiting underlying adherence effects on outcomes and allowing for a unique assessment of patients with limited health resource or medication access issues. Results suggest that among veterans initially adherent to their OADs those residing in the Northeast have comparatively better adjusted CAD and CVD outcomes than those in other regions. These were generally consistent by population density and among non-Hispanic Whites. Additionally, non-Hispanic Black veterans had lower hazards for CAD outcomes and all-cause mortality, which were consistent within population density and had little regional variation.

The combined inclusion of and controlling for race, region, and residential type is an important advancement in understanding how these factors contribute to disparate outcomes among Americans with diabetes. While earlier studies found significant racial, regional, and residential variation among patients with diabetes, these analyses either limited their focus to blood glucose levels or failed to control simultaneously for all three factors. Egede and colleagues observed small geographic variation and racial/ethnic differences in hemoglobin A1C levels, with lower levels among residents of the South and non-Hispanic Blacks; however, no urban/rural effects were uncovered. ${ }^{28}$ Similarly, Mainous and colleagues observed higher proportions of uncontrolled diabetes among African Americans, including substantial differences in proportions by urban/rural status. ${ }^{29}$ Collectively, these studies and evidence suggesting consistent differences in blood glucose levels by race/ethnicity suggest that minorities and, potentially, rural residents may be predisposed to worse diabetesrelated outcomes based on disproportionate disease management. ${ }^{30}$ However, our analyses, which further control for adequate medication use, seem to indicate that such

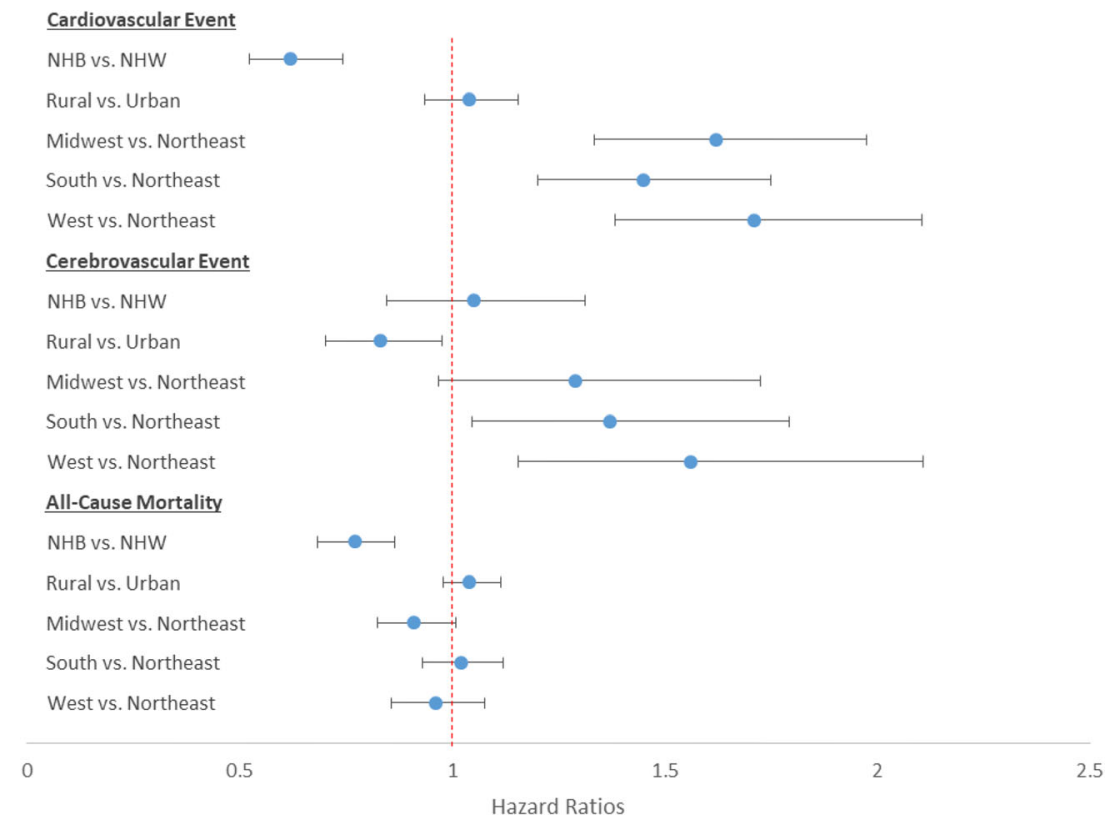

Figure 1 Adjusted hazard ratios for health outcomes within the first 5 years of oral antidiabetic medication use footnote: All models controlled for sex, marital status, age, CCI, median zip code income, baseline hemoglobin A1C, BMI, initial OAD, baseline systolic blood pressure, baseline LDL levels, whether the patient filled a lipid-lowering drug as of the first year of OAD use, and whether the patient filled an antihypertensive medication as of the first year of OAD use. Additionally, models controlled for record of a prior CAD or CVD event prior to initiating OAD treatment. 


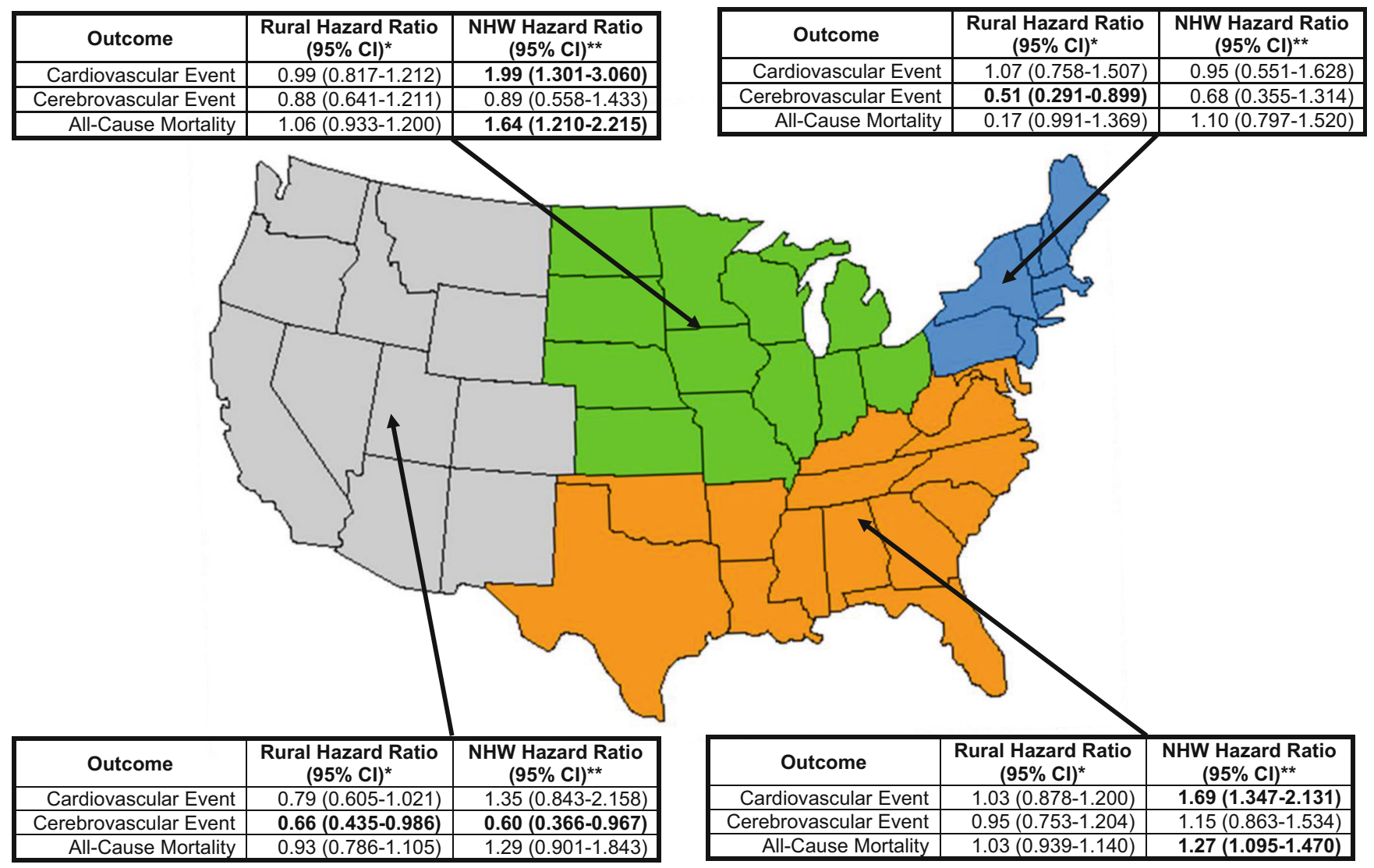

Figure 2 Within-region racial and residential differences in adjusted hazards for health outcomes within the first 5 years of oral antidiabetic medication use footnote: All models controlled for sex, marital status, age, CCI, baseline hemoglobin A1c, BMI, initial OAD, baseline systolic blood pressure, baseline LDL, and whether the patient filled an antihypertensive medication and/or a lipid-lowering drug as of the first year of OAD use. Additionally, models controlled for record of a prior CAD or CVD event prior to initiating OAD treatment. Interactions between region and race and urban/rural were included to determine within-region differences variation by race and residential type. Bolded values indicate statistical significance at $p<0.05$. * Compared to urban residents. **Compared to non-Hispanic Black veterans with diabetes. NHW: non-Hispanic White.

assumptions about relative disease burden are not empirically observed across outcomes. Consequently, our assessment builds off previous studies that incorporated race, region, and residential type into longitudinal assessment, but focused on discrete outcomes rather than intermediate measures of disease status. In doing so, and with respect to CVD outcomes and mortality by race, our findings mirror those of an earlier study among veterans. ${ }^{31,32}$

Among targeted subgroups, results suggest that little variation exists among non-Hispanic Black veterans for the included outcomes. However, significantly more variation may be present among non-Hispanic White veterans with results, in general, pointing towards more favorable outcomes in the Northeast. Perhaps unexpectedly, hazards for all three outcomes were lower or equivalent for non-Hispanic Black veterans even after adjusting for available covariates in both the Midwest and South, the latter a region in which disease control is reportedly worse. ${ }^{28,}{ }^{29}$ Proportionally, variation between regions was more prominent when compared to differences in outcomes within region (i.e., within-region differences by race and population density), suggesting that geography may play a relatively larger role in explaining disparate outcomes. However, within regions, at least in the case of CVD, the variation by race and population density may suggest some level of "neighborhood effect," the presence of which deserves further inquiry. ${ }^{33}$

Variations observed across outcomes and by race and geography provide further evidence of the need to examine underlying social determinants of health contributing to diabetes management. Our results echo an earlier call to identify both individual and combined influencers on diabetes outcomes across and within communities in all corners of the USA. ${ }^{34}$ By focusing on veterans with diabetes, we aimed to limit the impact of one pillar of the social determinants of health framework: healthcare access. ${ }^{35}$ A host of other factors within this framework remain to be examined and deserve inquiry to better address the needs of and reduce disparities among veterans and other Americans with diabetes.

This study was limited in several ways. The criteria required patients to have at least 1 year of follow-up after OAD initiation; therefore, the cohort includes only those who survived for at least that period. However, we believe the resulting immortal time bias was mild at best as our re-estimated all-cause mortality Cox model acknowledging late entry into the risk set did not show differences of practical importance. Survived cardiovascular and cerebrovascular events were observed during the first year as well (conditional on the patient surviving that first 
year) and are thus only indirectly affected by an immortal time bias as the patient had to survive 1 year, but the event of interest can occur earlier and would be recorded in that case. Secondly, data were limited to those in the VA electronic health records system and are subject to misclassification and other recording errors. Additionally, while PDC is a widely accepted adherence measure it remains an indirect metric. Moreover, the study did not incorporate longitudinal assessment of medication adherence; rather, we relied on our earlier findings that initial behavior was predictive of subsequent medication use and assumed that adherence would at least be similar beyond the first year. ${ }^{23}$ This may limit generalizability of findings to disparities surrounding those initiating therapy and may not be applicable to those who present with more advanced diabetes requiring insulin therapy upon diagnosis. Finally, this research was limited to veterans and results may not be generalizable to the broader US population.

In spite of these limitations, a key strength of this study is its use of a large electronic health record database from a closed healthcare system. By capturing nationwide VA patient records, nearly all encounters and drug fills can be incorporated. Consequently, analyses using these records can, with reasonable accuracy, identify the first diabetes diagnosis and OAD fill, allowing for longitudinal analysis from the identification of disease through treatment and outcomes.

Diabetes-related health outcomes remain likely among veterans in spite of achieving recommended antidiabetic treatment thresholds, and evidence suggests that racial and regional variation in cardiovascular or cerebrovascular events and all-cause mortality remain probable in such a subgroup. A deeper investigation of the role of social determinants of health will advance our understanding of local influencers on diabetes disease management.

Corresponding Author: Justin Gatwood, PhD; Department of Clinical Pharmacy University of Tennessee College of Pharmacy, 301 S. Perimeter Park Drive, Suite 220, Nashville, TN 37211, USA (e-mail:jgatwood@uthsc.edu).

Funders This study was supported by a KL2 Career Development grant from the University of Tennessee Health Science Center Institute for Research, Innovation, Synergy, and Health Equity, and by resources from the US Department of Veterans Affairs.

\section{Compliance with Ethical Standards:}

Institutional review boards at the University of Tennessee Health Science Center and the Memphis VA Medical Center approved this study.

Conflict of Interest: Csaba Kovesdy and Adriana Hung are employees of the Department of Veterans Affairs. Csaba P. Kovesdy received research grants from Abbvie, Bayer, and Janssen and served as consultant for Bayer. Justin Gatwood reports research grants from Merck \& Co. and GlaxoSmithKline. The remaining authors have no relevant conflicts to report.
Disclaimer: Opinions expressed in this presentation are those of the authors and do not represent the official opinion of the US Department of Veterans Affairs.

\section{REFERENCES}

1. Aikens JA, Piette JD. Longitudinal association between medication adherence and glycaemic control in type 2 diabetes. Diabet Med. 2013; 30(3): 338-344.

2. Lawrence DB, Ragucci KR, Long LB, Parris BS, Helfer LA. Relationship of oral antihyperglycemic (sulfonylurea or metformin) medication adherence and hemoglobin Alc goal attainment for HMO patients enrolled in a diabetes disease management program. J Manag Care Spec Pharm. 2006; 12: 466-471.

3. Horswell RL, Wascom CK, Cerise FP, Besse JA, Johnson JK. Diabetes mellitus medication assistance program: relationship of effectiveness to adherence. J Health Care Poor Underserved. 2008; 19: 677-686.

4. Adams AS, Trinacty CM, Zhang $\mathbf{F}$, et al. Medication adherence and racial differences in A1C control. Diabetes Care. 2008; 31: 916-921.

5. Rozenfeld Y, Hunt JS, Plauschinat C, Wong KS. Oral antidiabetic medication adherence and glycemic control in managed care. Am J Manag Care. 2008; 14: 71-75.

6. Kindmalm L, Melander A, Nilsson JLG. Refill adherence of antihyperglycaemic drugs related to glucose control (HbAlc) in patients with type 2 diabetes. Acta Diabetol. 2007; 44: 209-213.

7. Pladevall M, Williams LK, Potts LA, Divine G, Xi H, Lafata JE. Clinical outcomes and adherence to medications measured by claims data in patients with diabetes. Diabetes Care. 2004; 27: 2800-2805.

8. Nichols GA, Rosales AG, Kimes TM, Tunceli K, Kurtyka K, Mavros P. The change in HbAlc associated with initial adherence and subsequent change in adherence among diabetes patients newly initiating metformin therapy [published online August 7, 2016]. J Diabetes Res. 2016; doi: https://doi.org/10.1155/2016/9687815.

9. de Vries McClintock HF, Morales KH, Small DS, Bogner HR. Patterns of adherence to oral hypoglycemic agents and glucose control among primary care patients with type 2 diabetes. Behav Med. 2016; 42(2): 6371.

10. $\operatorname{Kim} \mathbf{N}$, Agostini JV, Justice AC. Refill adherence to hypoglycemic agents and glycemic control in veterans. Ann Pharmacother. 2010; 44(5): 800808 .

11. Krapek $\mathbf{K}$, King $\mathbf{K}$, Warren SS, et al. Medication adherence and associated hemoglobin Alc in type 2 diabetes. Ann Pharmacother. 2004; 38(9): 1357-1362.

12. Gatwood J, Chisholm-Burns M, Davis R, Thomas F, Potukuchi P, Hung A, Kovesdy CP. Differences in health outcomes associated with initial adherence to oral antidiabetes medications among veterans with uncomplicated Type 2 diabetes: a 5-year survival analysis. Diabet Med. 2018; 35(11): 1571-1579.

13. Curtis SE, Boye KS, Lage MJ, Garcia-Perez LE. Am J Manag Care. 2017; 23(7): e208-e214.

14. Han BH, Blaum CS, Ferris RE, Min LC, Lee PG. Older adults reporting more diabetes care have greater 9-year survival. J Am Geriatr Soc. 2015; 63(12): 2455-2462.

15. Zhu VJ, Tu W, Rosenman MB, Overhage JM. Nonadherence to oral antihyperglycemic agents: Subsequent hospitalization and mortality among patient with type 2 diabetes in clinical practice. Stud Health Technol Inform. 2015; 216: 60-63.

16. Fernandez A, Quan J, Moffet H, Parker MM, Schillinger D, Karter AJ. Adherence to newly prescribed diabetes medications among insured Latino and White patients with diabetes. JAMA Intern Med. 2017; 177(3): 371-379.

17. Agency for Healthcare Research and Quality, 2016 National Healthcare Quality and Disparities Report, (Rockville, MD: Agency for Healthcare Research and Quality, October 2017). Available at: https://www.ahrq. gov/sites/default/files/wysiwyg/research/findings/nhqrdr/ nhqdr16/final2016qdr-cx.pdf.

18. Kaiser Family Foundation. The Role of Medicaid in Rural America. Kaiser Family Foundation, Washington, DC, April 2017. Available at: https:// www.kff.org/medicaid/issue-brief/the-role-of-medicaid-in-ruralamerica. 
19. Kaiser Family Foundation. Disparities in health and health care: Five key questions and answers. Kaiser Family Foundation, Washington, DC, August 2018. Available at: https://www.kff.org/disparities-policy/issuebrief/disparities-in-health-and-health-care-five-key-questions-andanswers.

20. Chapko MK, Borowsky SJ, Fortney JC, et al. Evaluation of the Department of Veterans Affairs Community-Based Outpatient Clinics. Med Care. 2002; 40(7): 555-560.

21. VIReC Research User Guide: Veterans Health Administration Decision Support System Clinical National Data Extracts. 2nd Edition. Hines, IL: U.S. Dept. of Veterans Affairs, Health Services Research and Development Service, VA Information Resource Center; 2009.

22. Pharmacy Quality Alliance. PQA Performance Measures. 2016. https:// www.pqaalliance.org/pqa-measures. Accessed 21 Dec 2018.

23. Gatwood J, Chisholm-Burns M, Davis R, Thomas F, Potukuchi P, Hung A, Kovesdy CP. Disparities in Initial Oral Antidiabetic Medication Adherence among Veterans with Incident Diabetes. J Manag Care Spec Pharm. 2018; 24(4): 379-389.

24. U.S. Census Bureau. American FactFinder 2010 census. December 27, 2016. Available at: http://factfinder2.census.gov. Accessed December $21,2018$.

25. Deyo RA, Cherkin DC, Ciol MA: Adapting a clinical comorbidity index for use with ICD-9-CM administrative databases. J Clin Epidemiol. 1992; 45:613-619.

26. Chobanian AV, Bakris GL, Black HR, et al. Seventh report of the joint national committee on prevention, detection, evaluation, and treatment of high blood pressure. Hypertension. 2003; 42: 1206-1252.

27. Lin $\mathbf{D}$, Wei $\mathbf{L J}$, Ying Z . Checking the Cox model with cumulative sums of Martingale-based residuals. Biometrika. 1993; 80: 557-572.
28. Egede LE, Gebregziabher M, Hunt KJ, et al. Regional, geographic, and racial/ethnic variation in glycemic control in a national sample of veterans with diabetes. Diabetes Care. 2011; 34: 938-943.

29. Mainous AG, King DE, Garr DR, Pearson WS. Race, rural residence, and control of diabetes and hypertension. Ann Fam Med. 2004; 2(6): 563-568.

30. Campbell JA, Walker RJ, Smalls BL, Egede LE. Glucose control in diabetes: the impact of racial differences on monitoring and outcomes. Endocrine. 2012; 42: 471-482.

31. Young BA, Maynard C, Boyko EJ. Racial differences in diabetic nephropathy, cardiovascular disease, and mortality in a national population of veterans. Diabetes Care. 2003; 26: 2392-2399.

32. Kovesdy CP, Norris KC, Boulware LE, et al. Association of race with mortality and cardiovascular events in a large cohort of US veterans. Circulation. 2015; 132(16): 1538-1548.

33. Arcaya M, Tucker-Seeley $\mathbf{R}$, Kim $\mathbf{R}$, Schnake-Mahl A, So $\mathbf{M}$, Subramanian SV. Research on neighborhood effects on health in the United States: A systematic review of study characteristics. Soc Sci Med. 2016; 168: 16-29.

34. Walker RJ, Williams JS, Egede LE. Impact of race/ethnicity and social determinants of health on diabetes outcomes. Am J Med Sci. 2016; 351(4): 366-373.

35. Office of Disease Prevention and Health Promotion. Social Determinants of Health [Internet]. U.S. Department of Health and Human Services. Accessed 12 March 2019; available at: https://www.healthypeople.gov/ 2020/topics-objectives/topic/social-determinants-of-health.

Publisher's Note Springer Nature remains neutral with regard to jurisdictional claims in published maps and institutional affiliations. 